\title{
Effect of vegetation on flows and sediment transport
}

\author{
Hela Romdhane ${ }^{1}$, Amel Soualmia ${ }^{1, *}$, Ludovic Cassan ${ }^{2}$ and Gilles Belaud ${ }^{3}$ \\ ${ }^{1}$ National Institute of Agronomy of Tunisia, Water Science and Technology Laboratory, 43 Avenue \\ Charles Nicolle, 1082 Tunis, Tunisia \\ ${ }^{2}$ National Polytechnic Institute of Toulouse, Institute of Fluid Mechanics of Toulouse, Allée du \\ professeur Camille Soula, 314000 Toulouse, France \\ ${ }^{3}$ UMR G-eau, Montpellier Supagro, Univ. Montpellier, 2 Place Pierre Viala, 34060 Montpellier, \\ France
}

\begin{abstract}
Vegetation is a common feature in natural coastal and riverine waters, interacting with both water flow and sediment transport. However, the physical processes governing these interactions are still poorly understood, which makes it difficult to predict sediment transport and associated morphodynamics in a vegetated environment. In this context, an experimental study was conducted in laboratory with a movable bed trapped in artificial vegetation. The experimental flume is a rectangular open channel $5.75 \mathrm{~m}$ long and $0.29 \mathrm{~m}$ wide. For flow measurements, the channel is equipped with a fast camera and ADV probe. This work focuses on identifying the vegetation effects on flows and sediment transport. In fact, it was shown that the vegetation presence in a watercourse promotes deposition and sediment accumulation. This is explained by a reduction of the bed shear stress, since the friction occurs mainly by the drag force effect exerted by the vegetation. It was shown too that the vegetation reduced the bedload transport. Thanks to the partitioning of shear stress, it was possible to predict the bedload transport using standard formulas with a reasonable accuracy.
\end{abstract}

\section{Introduction}

The impact of vegetation on the sediment transport is a crucial issue to manage irrigation networks but also natural flows. By reducing velocity, the presence of vegetation may increase sediment deposition and modify the risk of flooding by the combined effects of increased roughness and decrease of flowing area of the river main channel [8]. In former studies, the role of the vegetation on hydraulic roughness was extensively investigated $[3,12,13]$. But there are fewer studies that link the velocity profile within the canopy to the sediment transport [2]. The aim of this paper is to show how a better understanding of the velocity field can help to understand the limitation of the sediment transport due to the presence of vegetation. To do so, measurements were performed with artificial vegetation in which a movable bed was installed. The analysis was carried out on

\footnotetext{
* Corresponding author: amel.inat@hotmail.fr
} 
velocity profiles and erosion dynamics within the vegetated bed. Velocity profiles will be compared to $1 \mathrm{D}$ vertical model because the validation of these models can bring information to understand the role of the vegetation on the sediment transport and elaborating a pertinent method of shear stress partitioning. In a second part, the experiments will emphasize the drastic influence of the vegetation on the solid rate, even if the hydraulic condition above canopy should involve sand movement.

\section{Material and Method}

\subsection{Experimental set-up}

Experiments were conducted in a flume $5.75 \mathrm{~m}$ long and $0.29 \mathrm{~m}$ wide. The bed was covered with artificial flexible vegetation made with thin circular cylinder of $0.8 \mathrm{~mm}$ diameter. A dozen of cylinders were gathered and stuck on a PVC blade at the same position. These basic vegetation cylinders were set in a staggered arrangement with $16.6 \mathrm{~cm}$ between stem. Considering an equivalent diameter of $4 \mathrm{~mm}$, the concentration is defined as $D^{2} / A$ where $A$ is the area around one stem $\left(A=2.7710^{-4} \mathrm{~m}^{2}\right.$ with $\left.m=3600 \mathrm{stems} / \mathrm{m}^{2}\right)$. The cylinder height is equal to $7 \mathrm{~cm}$. The spatial density which is equal to $m D=C / D$ remains almost constant in the vertical direction although near the canopy stems are no more contiguous.

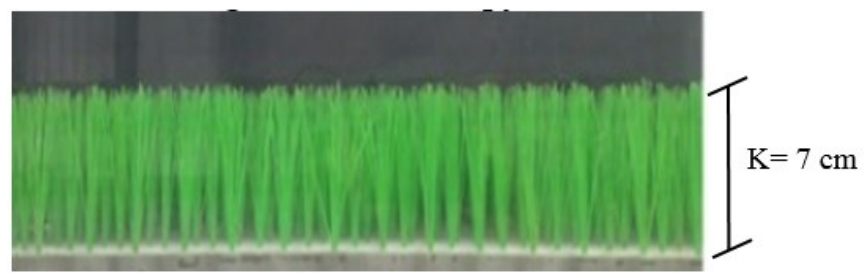

Fig. 1. Photograph of the used artificial vegetation.

The flume slope could be adjusted for 0 to a few percents. A weir at the upstream end allows fixing different water depths. The flow discharge was measured with an electromagnetic flowmeter with an uncertainty lower than $1 \%$.

A camera (Basler 5Mo, 100fps, 8 bits) was used to monitor the bed position. The size pixel is $1 \mathrm{~mm}$ and the resolution is $518 * 518$ pixels.

\subsection{Experimental procedure}

The velocity profile above the vegetation was obtained by Acoustic Doppler velocimetry with a micro-ADV Nortek Vectrino+ with a sampling rate equal to $25 \mathrm{~Hz}$. The vegetation was assumed to be dense enough to cause a velocity profile independent of the lateral position, even if, near the canopy, a slight discrepancy can be expected. The velocity measurements were performed for 2 flow rates and 2 slopes.

The sediment was a mix of sands and glass (for pool filter). The measured density $\left(\rho_{s}\right)$ and porosity $\left(p_{0}\right)$ were respectively equal to $2350 \mathrm{~kg} / \mathrm{m}^{3}$ and 0.6 . The grain size distribution was obtained with a mechanical shaker and sieves (see grading curve in Fig. 2). At the beginning of each experiment, the vegetation was totally recovered by the sand $(\sim 5 \mathrm{~mm}$ above top of canopy) in a zone of 1 meter long at the flume center (figure 3 ). The tail weir was set high enough to impose a high-water depth and limit the wall shear stress. The flow was maintaining during a few minutes until steady flow and stable bed were obtained. Then, in order to increase the bed shear stress, the weir was moved down by steps of $1 \mathrm{~cm}$, until the weir was totally removed. 

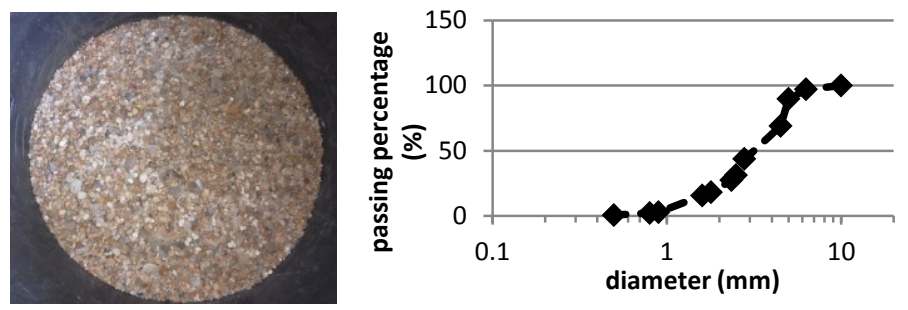

Fig. 2. Photograph of the sediments (left) and grain size distribution (right).

These experiments were done with the flow rate of $14.5 \mathrm{l} / \mathrm{s}$, and with two different slopes of $0.3 \%$ and $1 \%$ (table 1 ).

Table 1 : Experiments with sediments

\begin{tabular}{|c|c|c|}
\hline & $\mathbf{S = 0 . 3 \%}$ & $\mathbf{S}=\mathbf{1 \%}$ \\
\hline Flow rate (l/s) & 14.5 & 14.5 \\
\hline upstream water depth (cm) & 20.5 & 20.5 \\
\hline downstream water depth (cm) & 21 & 21 \\
\hline Initial weir position (cm) & 14 & 14 \\
\hline Length of Vegetation layer (m) & 4 & 4 \\
\hline Length of Sediment layer (m) & 1 & 1 \\
\hline Duration of experiment (h) & 10 & 9 \\
\hline
\end{tabular}
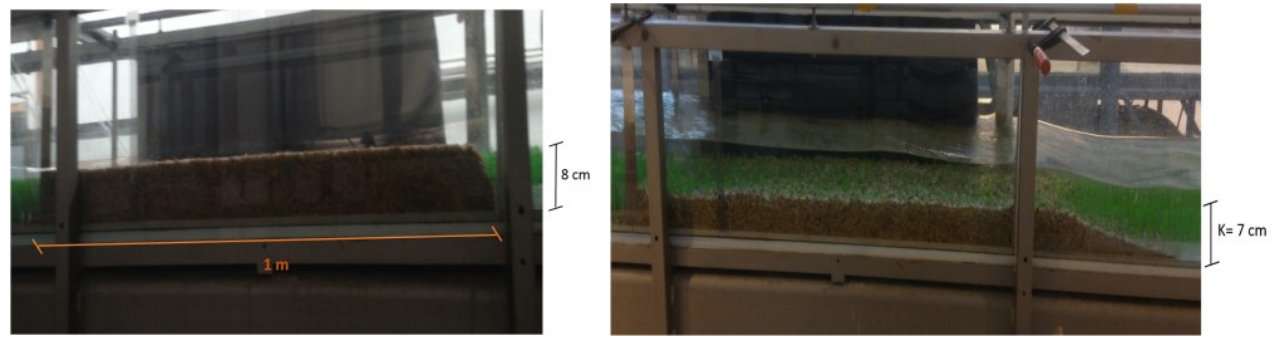

Fig. 3. View of the bed before (left) and during the end of experiment (right) with $\mathrm{S}=1 \%$.

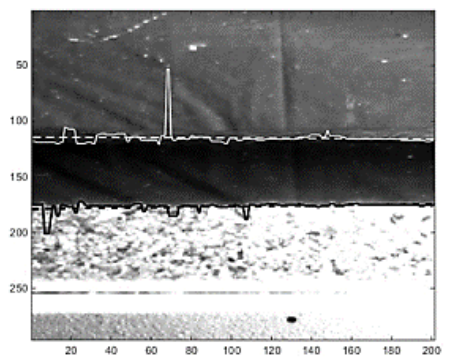

Fig. 4. picture showing the bed position and water free surface at time $t=12000 \mathrm{~s}(\mathrm{~S}=1 \%)$. The solid lines represent the detected interface; the dashed lines are obtained by linear regression used to find the averaged positions. 


\section{Results}

\subsection{Velocity profiles without sediment}

The ADV velocity profiles were compared to the velocity distribution as proposed in [7] and DBM models [1,9]. These models give the velocity within and above the vegetation based on turbulence length scale considerations. The experimental correlation provides the turbulent length scale in the canopy, while the velocity distribution is assumed to follow a logarithmic function above the canopy up to free surface. In [9], the experimental correlation is substituted by another model based on the distance $(s)$ between stems, and the stem height $k$. In [7], the length scale is also assumed to be equal to $s$ but the dimensional analysis only provides the average velocity within the canopy $U_{r}(\mathrm{~m} / \mathrm{s})$ and above the canopy $U_{s}(\mathrm{~m} / \mathrm{s})$, and the resulting model consists of uniform velocities within the canopy, and above the canopy.

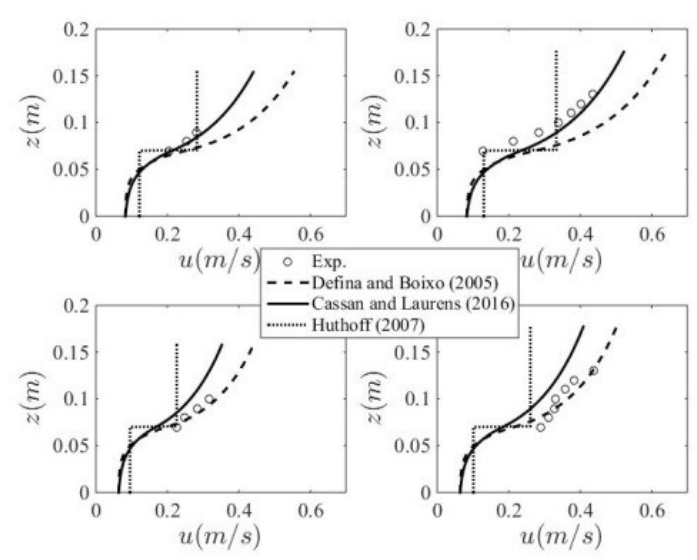

Fig.5. Vertical velocity profile from ADV, and model from Defina and Boixo [1], Hutoff [7] and Cassan and Laurens [9].
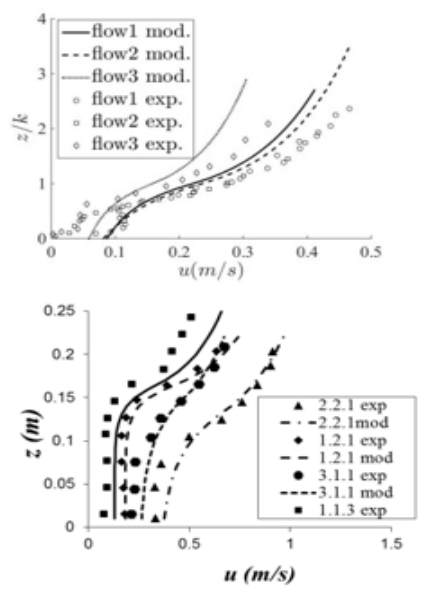

Fig.6. Comparison between velocity of [2] (top), [4] (down) and Cassan and Laurens model for flexible vegetation.

Clearly, both DBM models ([1,9]) are more relevant to describe the velocity distribution in the upper layer even. Within the canopy, the predicted velocity is close to the average value, as predicted by Hutoff model (Fig. 5). To verify the ability to reproduce the velocity distribution in a flexible canopy, we compared the model of [9] to the experimental results of [2] and [4]. The good agreement as shown in Figure 6 confirms the validity of the model already, as found in previous experimental studies [9].

We will now use the above models to analyze the prediction of the bed shear stress.

\subsection{Bed shear stress}

The DBM models provide the distributed turbulent shear stress along the vertical axis. However, this friction is independent of the bed roughness and is only due to the drag dissipation and flow above the roughness. Moreover, since the turbulent length scale is calibrated from the characteristics of the flow near the top of the canopy, there is no reason that it would correctly represent the flow near the bed. 
Alternatively, one could use bed friction laws, such as Manning friction loss equation [7] or other friction laws [10]. However, this would require considering the average velocity and friction coefficients calibrated from experiments without vegetation. We expect that, in absence of vegetation, the boundary layer largely differs from the one in presence of stems (for instance due to HorseShoe Vortex HSV). We proposed here to consider a second approach which has the advantage to be consistent with Manning's friction equation.

As a consequence, the bed shear stress can be approximated by [7]:

$$
\tau_{b}=\rho f U_{r}^{2}=\rho \frac{1}{64}\left(\frac{k_{s}}{h}\right)^{1 / 3} U_{r}^{2}
$$

Where $f$ is the friction coefficient, $k_{s}(\mathrm{~m})$ is the height of the irregularities at the bed and $h$ the water depth. The bed shear stress can be computed with the velocity near the bed, but for sparse vegetation and small stem diameter, the turbulence due to the above layer can penetrate into the canopy, down to the bed (figure 7). Therefore, we assume that the bed shear stress is modelled with the average velocity $U_{r}$ in the bed [7], but we use the velocity distribution to improve the computation of the drag term. In a recent paper [2] compared several methods to compute the shear stress partitioning in a vegetated flow. The Einstein partitioning method was the most appropriate approach to describe parameter $\alpha$ defined as the ratio between the bed shear stress and the total shear stress $\tau_{\tau o \tau}\left(\tau_{\tau o \tau}=\rho g S h\right)$. Our method is a combination of the Einstein method to compute the friction coefficient and the explicit formulation to evaluate the reference velocity $\left(U_{r}\right)$.

Then the momentum balance in the canopy can be rewritten, for a bed surface $A$ around one stem:

$$
\begin{gathered}
\left(\rho f A U_{r}^{2}+\frac{1}{2} \rho \beta C_{d} D k\right) U_{r}^{2}=g S A k\left(\frac{h}{k}-\sigma C\right) \\
U_{r}^{2}=\frac{2 g S\left(\frac{h}{k}-\sigma C\right)}{\beta C_{d} \frac{C}{D}+\frac{f}{k}}
\end{gathered}
$$

With: $\sigma$ is the shape factor $(=\pi / 4), g$ the gravitational constant, $C_{d}$ the stem drag coefficient, and $\beta$ is equivalent to a Boussinesq factor defined as:

$$
\beta=\frac{1}{k U_{r}^{2}} \int_{0}^{k} u^{2} d z
$$

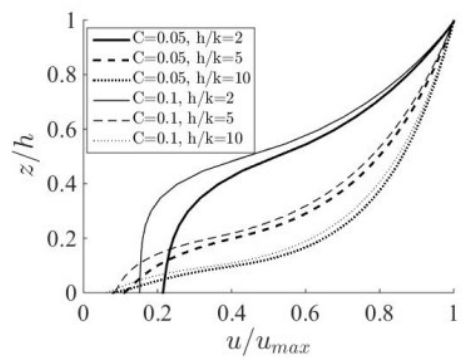

Fig. 7. Velocity profile with DBM [9], for $\mathrm{D}=0.004 \mathrm{~m}$.

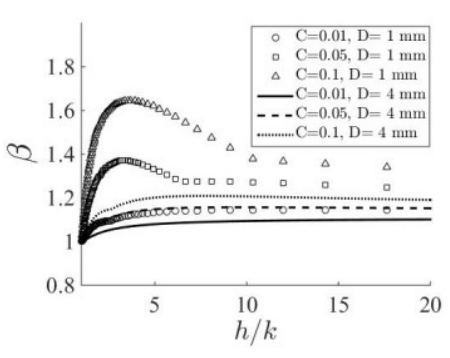

Fig. 8. Momentum parameter coefficient as a function of the submergence for $\mathrm{h}=15 \mathrm{~cm}$.

The averaged velocity, $U_{r}$, is obtained by integrating the velocity distribution. When the velocity profile is almost uniform, as assumed by Hutoff et al. [7], $\beta$ tends to 1 . This case is only valid for sufficient dense and high vegetation (figure 7 ). For the present 
experimental case $(h=15 \mathrm{~cm})$, figure 8 shows that $\beta$ can vary from 1.1 to 1.6 for thicker stems. Coefficient $\beta$ is constant for large $h / k$. This behavior occurs when the turbulent length scale equal to $0.15 k$ [9] which corresponds to our experimental cases. In the following, it is considered that $\beta=1.2$.

Combining equations 1,2 and 3 , it is possible to express $\alpha$ for submerged conditions:

$$
\alpha=\frac{\tau_{b}}{\tau_{\text {total }}}=\frac{\left(1-\sigma C \frac{k}{h}\right)}{2 \beta \frac{C_{d} C k}{f D}+1}
$$
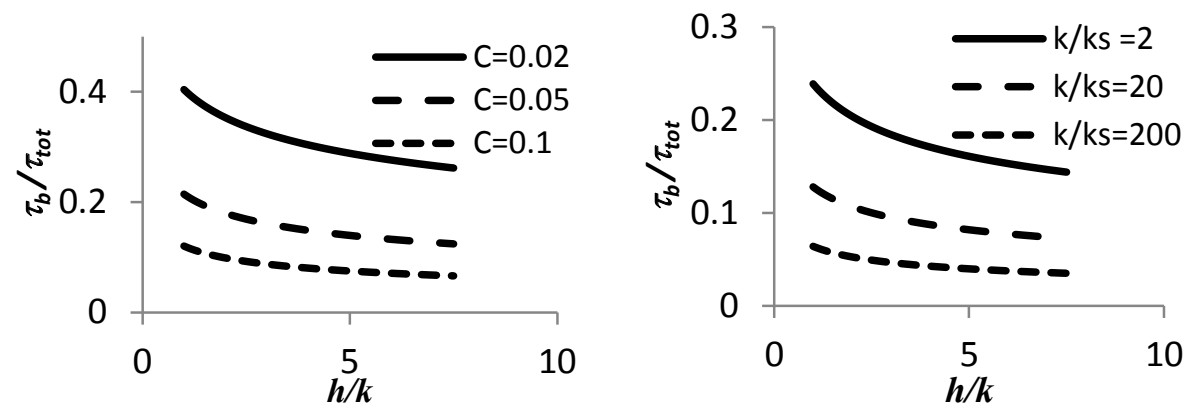

Fig. 9. Evolution of the coefficient $\alpha$ as a function of the submergence for various bed grain size with $C=0.05$ (left) and various density with $k / k_{s}=10$ (right). $k / D=0.005 / 0.004=1.25$ for both graphs corresponding to our experiments.

The shear stress partitioning $\alpha$ is computed for various configurations of vegetated bed (Fig. 9). For our experiments, where $\mathrm{C}=0.05$ and $k / k_{s} \sim 2$, the model gives $\alpha=0.2$. For the same grain size, when the vegetation height increases, $\alpha$ decreases. Then, when $k / k_{s}$ is 100 times larger, $\alpha$ drops to $5 \%$ and less. Note that in [2], erosion could be observed even when $k / k_{s}=1000$. This could be explained by HSV which is not taken into account here. The partitioning from the model lso become very low (less than 10\%) when the density is greater than 0.01 .

\subsection{Sediment rate in experiments}

The water surface profile was measured in order to determine the slope friction. The kinetic energy was neglected, and a uniform value of the slope is obtained by considering the difference of the water depth $(\delta h)$ at $\mathrm{X}=2.5$ and $\mathrm{X}=3.5$ (figure 10). The water depth variation $(\delta h)$ was then added to the bottom slope in order to compute the total head loss, and then the bed shear stress (Eq.1). The $\delta h$ value has the same order of magnitude that the bed slope, i.e between $(-0.5$ and $2 \%)$.

Figure 10 gives the evolution of the water depth and bed during the both experiments. The bed reference corresponds to the top of the canopy. When the vegetation is totally covered, the bed is eroded with an almost constant rate even if the shear stress increases slowly due to weir movement. When the vegetation progressively appears, due to sand entrainment, the erosion rate decreases drastically, which highlights the strong influence of vegetation on the bed shear stress and then sediment erosion. 

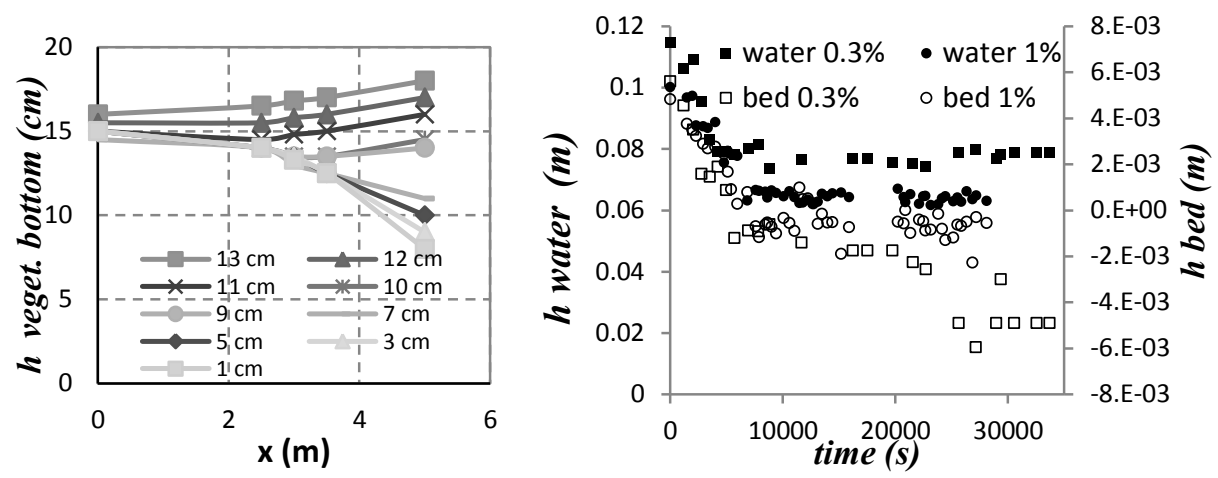

Fig. 10. Water surface profile from vegetation bottom during the sediment experiments (left); average water distance from top of canopy and bed position at the camera measurement $(X=3 \mathrm{~m})$ (right).

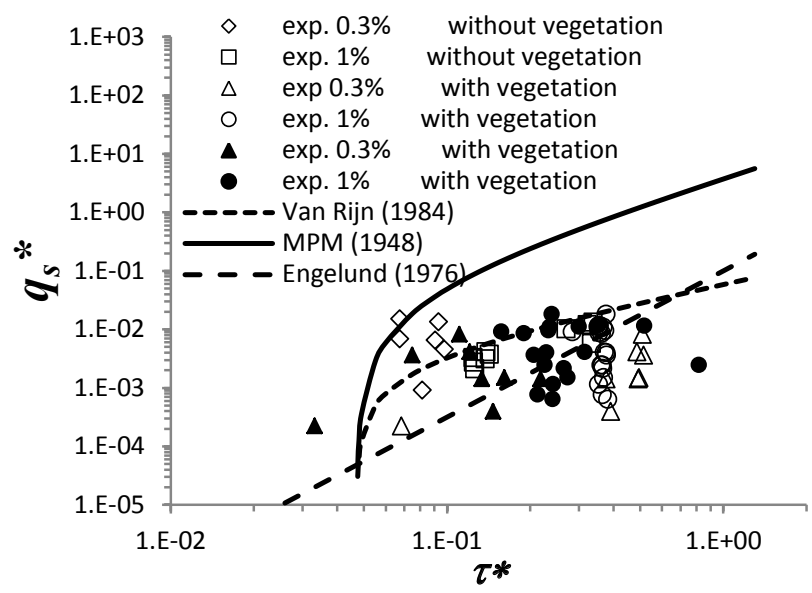

Fig. 11. Solid rate as a function of the normalized shear stress. Measurement without vegetation means that the bed is due to sand because vegetation was still totally covered $\left(\tau_{\text {total }}=\tau_{\text {bed }}\right)$.

The volumetric solid discharge by unit width $q_{s}$ was evaluated by calculation of the bed sediment volume (on the length of $1 \mathrm{~m}$ ) at each time. Since no sediment is incoming in the control volume, $q_{s}$ is obtained directly from the volume variation with respect to time, which is the Exner equation:

$$
q_{S}^{*}=\frac{q_{s}}{d_{50} \sqrt{\frac{\rho_{s}-\rho}{\rho} g d_{50}}}=\frac{\left(1-p_{0}\right) \frac{d h_{\text {bed }}}{d t} L}{d_{50} \sqrt{\frac{\rho_{s}-\rho}{\rho} g d_{50}}}
$$

The experimental normalized sediment rate $q_{s}{ }^{*}$ is compared to classical bed load transport formulas (Fig. 11). Before the vegetation appears, the experimental value of $q_{s}{ }^{*}$ is consistent with Van Rijn [11] and Engelund and Fredsoe's [6] formulas. The Meyer Peter Muller formula overestimates the bedload but note that in this case $d_{50}$ is out of the range of validity. On the other hand, when the vegetation is discovered, the measured sediment rate is quite lower than the calculated one for the normalized shear stress greater than 0.5 . As expected, the vegetation reduces the transport rate, which we can explain by the decreased ratio $\alpha$ of bed shear stress to total shear stress. Using equation $5, \tau^{*}=\tau /\left(\rho_{s}-\rho\right) g d_{50}$ is recomputed to represent more precisely the flow near the bed. With $\beta=1.2$, the new computed bed shear stress $\left(\tau_{b}=\alpha \tau_{t o t}\right)$ improves the estimation of $q_{s}{ }^{*}$ (Fig. 11, $\tau_{b}$ with vegetation). 
Therefore, this reinforces the interest of using a model taking explicitly into account the effect of vegetation on flow properties. The model presented here allows to correct existing bedload formulas by considering explicitly the effect of vegetation, through the correction ratio $\alpha$.

\section{Conclusion}

Experiments were conducted in laboratory in order to show the effect of vegetation on velocity profiles and non-cohesive sediment erosion. The presence of vegetation induced a large decrease of bed shear stress, and therefore strongly limited the bed erosion. A method to compute the shear stress partitioning in presence of vegetation was developed here, based on the description of the vertical velocity distribution and Manning friction law. On the one hand, the method could give an accurate prediction of the velocity profile. On the second hand, it allowed calculating the shear stress decrease due to vegetation, as a function of the vegetation height and density. This correction is a way to consider the effect of vegetation on sediment transport prediction. By doing so, standard sediment transport formulas gave a reasonable prediction of observed sediment rates.

\section{References}

1. A. Defina, A. Bixio, Mean flow and turbulence in vegetated open channel flow. Water Ressour. Res. 41 (2005).

2. C. Le Bouteiller, J.G. Venditti, Vegetation-driven morphodynamic adjustments of a sand bed, Geophys. Res. Lett., 41 (2014).

3. D. Poggi, C. Krug, G.G. Katul, Hydraulic resistance of submerged rigid vegetation derived from first-order closure models, Water Resour. Res., 45 (2009).

4. E. Kubrak, J. Kubrak, PM. Rowinski, Vertical velocity distributions through and above submerged, flexible vegetation. Hydrol Sci J, 53, 4 (2008).

5. E. Meyer-Peter, R. Müller, Formulas for bed load transport, paper presented at 2nd Meeting of International Association for Hydraulic Research, Int. Assoc. for Hydraul. Res., Stockholm. (1948).

6. F. Engelund, and J. Fredsoe, A sediment transport model for straight alluvial channels, Nord. Hydrol., 7, 5 (1976).

7. F. Huthoff, D.C.M Augustijn, S. J. M. H. Hulscher, Analytical solution of the depthaveraged flow velocity in case of submerged rigid cylindrical vegetation, Water Resour. Res., 43 (2007).

8. H. Romdhane, A. Soualmia, L. Cassan, Modelling and Analysis of the Hydrodynamic of the Medjerda River- Impacts of Dredging. Tunisia-Japan Symposiumon Science, Society and Technology (TJASSST), Gammart, Tunisia, (2017).

9. L. Cassan, P. Laurens, Design of emergent and submerged rock-ramp fish passes. Knowl. Manag. Aquat. Ecosyst., 417, 45 (2016).

10. L. Cassan, H. Roux, PA. Garamboix, A semi analytical model for the hydraulic resistance due to Macro-roughness of varying shapes and densities, Water, 9, 637 (2017).

11. L. Van Rijn, Sediment Transport, Part 1-2-3: Bed Load Transport, J. Hydraulic Eng. 110, 10 (1984).

12. M. Luhar, J. Rominger, H. Nepf, Interaction between flow, transport and vegetation spatial structure. Environ. Fluid Mech. 8 (2008).

13. N. Nikora, V. Nikora, T. O’Donoghue, Velocity profiles in vegetated open-channel flows: combined effects of multiple mechanisms. J Hydraul. Eng., 139, 10 (2013). 